\title{
Efeitos de exercícios para o sistema vestibular na melhora da tontura e do equilíbrio após acidente vascular encefálico
}

\author{
Effect of exercise in the vestibular system in the symptom \\ on increase dizziness and balance after stroke
}

\author{
Andelize Bonadiman ${ }^{1}$ \\ Leandro Caetano Guenka² \\ Joeli Vedana ${ }^{3}$
}

\section{RESUMO}

O acidente vascular encefálico (AVE) afeta a função dos sistemas sensoriais ocorrendo discrepância nas informações provenientes do sistema vestibular (SV). O SV é responsável pelo equilíbrio sendo resultado da interação de diversos sistemas que mantém o corpo contra a ação da gravidade, ao haver complicações o paciente terá queixas de vertigens, tonturas e déficit de equilíbrio. A reabilitação vestibular (RV) tem como objetivo restabelecer a manutenção do equilíbrio e sintomas associados por meio de exercícios baseados em adaptação, habituação e substituição. O objetivo deste estudo foi verificar o efeito de exercícios de RV, juntamente com treino de equilíbrio sobre a vertigem e o desequilíbrio após AVE. A amostra foi constituída por 10 pacientes adultos com idade de 50 a 70 anos, com diagnósticos de acidente vascular encefálico isquêmico ou hemorrágico com tempo de lesão em média de 5 anos. Foi utilizado a Escala de equilíbrio de Berg de Dizziness Handicap Inventory (DHI). Foram observadas melhoras significativas na diminuição dos sintomas de tontura no DHI com média inicial de 49, 2 pontos e a final de 38,2 ( $P=0,001)$ e na Escala de Equilíbrio de Berg com média inicial de 59 e final de 66 pontos $(P=0,03)$. Os exercícios utilizados de reabilitação vestibular foram relevantes na melhora nos sintomas de tontura e no incremento do equilíbrio, possivelmente através de mecanismos de adaptação, habituação e compensação que induzem a uma neuroplasticidade no sistema nervoso.

\section{PALAVRAS-CHAVE}

Acidente vascular encefálico; Equilíbrio. Sistema vestibular; Tontura.

\footnotetext{
${ }^{1}$ Graduada em Fisioterapia pela UNISEP/FEFB.

${ }^{2}$ Professor do Curso de Fisioterapia da UNISEP/FEFB.

${ }^{3}$ Professora do Curso de Fisioterapia (UNISEP/FEFB).
} 


\section{ABSTRACT}

Stroke affects the function of the sensory systems, with discrepancy in the information coming from the vestibular system (SV). The SV is responsible for the balance being the result of the interaction of several systems that maintains the body against the action of gravity, when there are complications the patient will have complaints of vertigo, dizziness and balance deficit. Vestibular rehabilitation (VR) aims to reestablish the maintenance of balance and associated symptoms through exercises based on adaptation, habituation and substitution. The purpose of this study was to verify the effect of VR exercises, along with balance training on vertigo and imbalance after stroke. The sample consisted of 10 adult patients aged 50 to 70 years, with diagnoses of ischemic or hemorrhagic encephalic vascular accident with an average injury time of 5 years. The Berg Balance Scale of Dizziness Handicap Inventory (DHI) was used. Significant improvements were observed in the reduction of the symptoms of vertigo in the IHD with an initial mean of 49, 2 points and the final of 38.2 (P $=0.001)$ and in the Berg Balance Scale with initial mean of 59 and final of 66 points $(P=0.03)$. The exercises used for vestibular rehabilitation were relevant in improving the symptoms of vertigo and increasing the balance, through mechanisms of adaptation, habituation and compensation that induce neuroplasticity in the nervous system.

\section{KEYWORDS}

Stroke; Balance; Vestibular system; Dizziness. 


\section{INTRODUÇÃO}

As consequências após AVE podem ser caracterizadas por dificuldade em manter a posição ocular normal, alteração na mobilidade ocular, estrabismo e ou nistagmo e dificuldade na realização de movimentos oculares espontâneos e voluntários (POLLOCK et al., 2011). Assim, como modificações no sistema vestibular podem apresentar instabilidade postural, disfunção de equilíbrio, alteração na marcha, risco de queda consequentemente diminuição na funcionalidade e prejuízo na realização de atividades de vida diária (GRILL et al., 2013; WHITNEY et al., 2016. A vertigem pode estar presente em pacientes com AVE devido ao fato de existir a lesão de hemisférios cerebrais, pois é considerado que lesões vestibulares do tronco encefálico e do cerebelo são capazes de provocar a vertigem (PIRES et al., 2013).

Exercícios realizados na reabilitação vestibular têm como foco o treino postural, para tratar não apenas as instabilidades, mas também vertigem e tonturas. Estes treinos estimulam o sistema nervoso periférico aumentando a integração com sistema nervo central, fornecendo inputs sensoriais espaciais mais adequados, para correção do alinhamento da cabeça e do corpo (TJERNSTRO"M et al., 2016). Quando são realizados testes de AVD's e avaliação do equilíbrio em paciente que sofreram AVE podemos perceber, que entre as queixas apresentadas pelos indivíduos, está o déficit no equilíbrio e sintomas de vertigem e tontura. $\mathrm{O}$ sistema vestibular é um dos principais, junto com o cerebelo, responsáveis pelo controle e manutenção do equilíbrio (SALES, 2013).

Portanto, exercícios específicos podem melhorar o sistema somatossensorial a interação vestíbulo visual durante a movimentação cefálica, com a utilização de diferente superfície de suporte e com os olhos fechados (Ricci et al., 2010). Consequentemente, o objetivo deste trabalho foi verificar os feitos de exercícios para o sistema vestibular na melhora da tontura e do equilíbrio após acidente vascular encefálico.

\section{MATERIAIS E MÉTODOS}

Foi realizado um estudo experimental do tipo transversal, com uma amostra de 10 pacientes adul- tos, com idade entre 50 e 70 anos, tempo de lesão em média de 5 anos, com diagnóstico de acidente vascular encefálico isquêmico ou hemorrágico. Forma selecionados através da análise de prontuários pertencentes ao Posto de saúde de Campo Erê, Santa Catarina, no qual os selecionados deveriam ter diagnóstico de Acidente Vascular Encefálico. Os critérios de inclusão foram ter sofrido AVE hemorrágico ou isquêmico, que possuíssem deambulação independente ou com auxilio, apresentando vertigem e desequilíbrio.

Os critérios de exclusão foram que apresentassem hipertensão arterial não controlada, deambulação incapaz, patologias neurológicas associadas ou musculoesqueléticas que impossibilitam o paciente a realizar os exercícios de Cawthorne e Cookse, exercícios de Herdman e as manobras de nistagmo. Esta pesquisa foi realizada na cidade de Campo Ere do estado do Santa Catarina no período de agosto a outubro no ano de 2015. Os participantes concordaram e assinaram o Termo de Consentimento Livre e Esclarecido. Esta pesquisa foi aprovada pelo Comitê de Ética em Pesquisa da UNISEP/FEFB, com CAAE da Plataforma Brasil ( ${ }^{\circ}$ 46108415.7.0000.5230).

Os participantes responderam no início e no final do tratamento o questionário de tontura Dizziness Handicão Inventory (DHI) brasileiro (CASTRO et al., 2007) e o questionário de equilíbrio pela escala de Berg (MIYAMOTO, 2004). No questionário DHI na versão brasileira o maior escore total obtido máximo é de 100 pontos, situação em que se observa um maior prejuízo causado pela tontura; e o menor, zero ponto, que revela nenhum prejuízo devido à tontura. Já a Escala de Equilíbrio de Berg a pontuação máxima é de 56 pontos, situação em que se observa melhor desempenho do paciente no equilíbrio, e quanto menor for a pontuação maior será o risco para quedas.

No exame físico foi testado o equilíbrio dinâmico, sendo realizado o teste Babinski-Weil (SILVA; MOREIRA, 2000), manobra de Brandt-daroff e manobra de Thrust cefálico. No tratamento foram feitas a manobra de Semont (ALBERTINO, 2012) complementando com exercícios de Cawthorne \& Cookse e de Herdman (RIBEIRO, 2005) para tratar a vertigem e adaptar o sistema vestibular auxiliando no equilíbrio e no 
reflexo RVO, realizado 3 vezes por semana totalizando 10 sessões, em média de 40 minutos a sessão. Ainda, o estudo segui algumas recomendações de Lacour e Demanze (2014), para incrementar a recuperação funcional como: ser cuidado com a progressão do tratamento, reduzir ansiedade e estresse, contexto e situações ambientais favoráveis, motivação do paciente

O teste utilizado para análise estatística dos dados foi o teste $t$, após a verificação da normalidade das variáveis pelo teste de Shapiro-Wilk. O programa utilizado foi o SPSS 22.0, Windows. Com significância estatística de $5 \%$.

\section{RESULTADOS}

Observamos no DHI que a média inicial foi de 49, 2 pontos e a final foram de 38,2 e desta maneira obteve-se uma média de melhoria de 11 pontos na relação entre a média inicial e final. Apresentando diferença estatisticamente significante $(P=0,001)$.

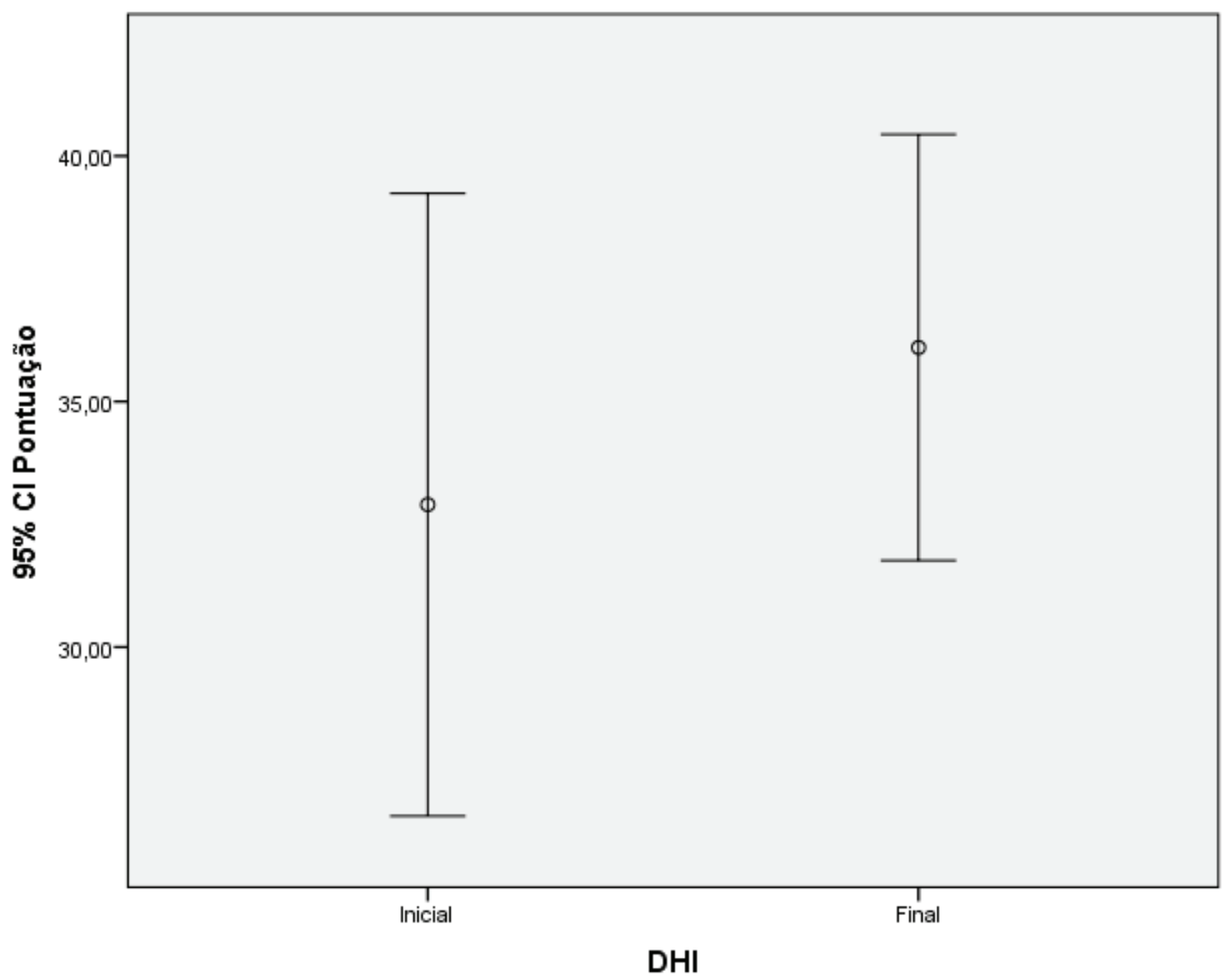

Figura 1: Comparação entre as médias, do questionário de DHI.

Em relação a Escala de Equilíbrio de Berg também apresentou diferença estatística no equilíbrio dos pacientes após a reavaliação de $(P=0,03)$, onde a média inicial dos pacientes foi de 59 inicial e a final foi de 66 totalizando 7 pontos de melhora. 


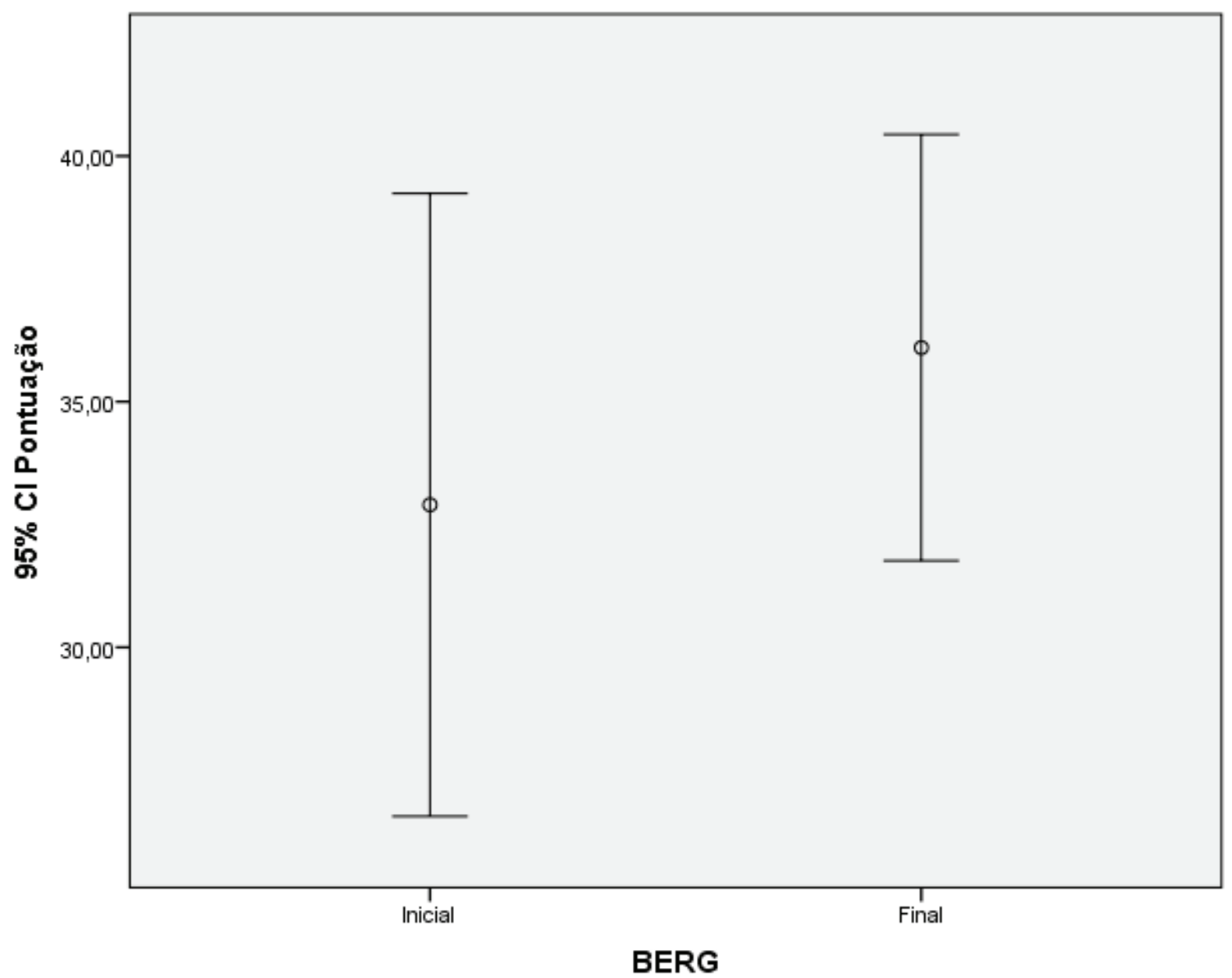

Figura 2: Comparação entre as médias, da Escala de Equilíbrio de BERG.

\section{DISCUSSÃO}

A reabilitação vestibular (RV) é um tratamento que apresenta resultados para pacientes com AVE e persistência da vertigem devido à sua disfunção, proporcionando melhora no equilíbrio e diminuição da vertigem. As alterações do equilíbrio se originam de afecções vestibulares, centrais ou periféricas, proprioceptivos e de várias vias de integração destes com o restante do SNC. O AVC acomete o SNC, contribuindo para o desenvolvimento de síndromes vestibulares, que de forma frequente cursam com alterações do equilíbrio (SIMONEAU et al., 1995; PAVAN et al., 2000).

Diante do objetivo do presente estudo podemos verificar, que após 10 sessões de tratamento, houve uma diminuição significativa dos sintomas de vertigem e desequilíbrio com o uso da RV, proporcionan- do a melhora na qualidade de vida e favorecendo as AVD's. Sabendo que esse tratamento foi baseado na reabilitação de mecanismos da plasticidade neuronal do sistema nervoso, que tem como objetivo a ativação desses mecanismos, específicos e repetitivos, levando a um reequilíbrio dos núcleos vestibulares com adequações sinápticas (TJERNSTRO"M et al., 2016; GANANÇA et al., 2004). Nessa adaptação o sistema vestibular foi adaptado a receber e processar as informações que nele chegaram mesmo incompletas, adequando aos estímulos para responder de forma correta, para que as informações relacionadas ao sintoma de equilíbrio e vertigem que estão ausentes nesses indivíduos sejam substituídas (ALBERTINO, 2012).

Wyk et al., (2014), verificou no seu ensaio clínico randomizado, que após 4 semanas de exercícios visuais durante atividades especificas em participante 
com lesão no córtex parietal posterior, pós AVE apresentaram resultados significativos, na melhora da atividade funcional, com um incremento no sistema vestibular e somatosensorial, controle e manutenção postural durante movimentos funcionais. $O$ estudo de Pires et al., (2013) confirma que pacientes pós $A V E$, podem apresentar tontura ou desequilíbrio corporal (72,5\%), na fase tardia e sinais de comprometimento da motilidade ocular e da função vestibular, comprovados após avaliar a função vestibular de 40 pacientes, por meio de exame otorrinolaringológico, questionário $\mathrm{DHI}$ e vectoeletronistagmografia. Outro estudo também empregou o DHI para avaliar a qualidade de vida em relação à tontura, antes e após a intervenção, por meio dos exercícios de reabilitação vestibular, constataram uma melhora após o tratamento (MANTELLO et al., 2008).

Toledo et al., (2000) comparou a eficácia da manobra de Semont, com exercícios de reabilitação vestibular no tratamento da vertigem posicional paroxística benigna (VPPB) durante 3 meses em 40 pacientes, verificou que a manobra de Semont apresentou melhoras após 2 meses de tratamento. No estudo de Fernandes e Vasconcelos (2012) foi realizado um estudo de casos com 5 pacientes após AVC avaliados pela Escala Modificada de Rankin (MRS), Quociente de Sensibilidade Motora (QSM), Escala de Equilíbrio de Berg (BBS), Escala de Equilíbrio e Mobilidade de Tinetti (TBMS), Escala Específica de Qualidade de Vida (SS-QOL) para AVC e a versão Brasileira do Dizziness Handicap Inventory (DHI). O protocolo de tratamento foi baseado nos exercícios de Cawthorne e Cooksey da RV revelaram uma melhora estatisticamente significante no equilíbrio, mobilidade e qualidade de vida. Porém não houve redução significante do índice de vertigem (DHI).

O objetivo principal das manobras de reabilitação vestibular como a manobra de Semont, é recolocar os debris livres do(s) canal(is) semicircular(es) para o utrículo e o sáculo, onde eles tendem a aderirem. Os exercícios buscam alcançar mecanismos de adaptação e compensação no sistema nervoso central, visando à superação dos sintomas (TEIXEIRA; MACHADO 2006).

Este estudo tem como limitações, a falta de provas de função vestibular, como nistagmografia
(ENG), vectoeletronistagmografia (VENG) e posturografia. E ausência de comparação de comparação com um grupo controle. Porém, a partir dos resultados de nossa pesquisa, avaliados por meio do questionário DHI e da escala de equilíbrio de BERG, observou-se uma melhora tanto em relação à tontura quanto na funcionalidade após a intervenção e um incremento no equilíbrio, o que contribuíram para uma vida mais independente e ativa.

\section{CONCLUSÃO}

Os exercícios utilizados de reabilitação vestibular, associados a manobra de Semont, foram relevantes na melhora nos sintomas de vertigem e no incremento do equilíbrio, através de mecanismos de adaptação, habituação e compensação que induzem a uma neuroplasticidade no SN, levando a recuperação funcional dos desequilíbrios corporais. Desta forma, através deste estudo, conseguimos notar que um trabalho com enfoque na melhora dos mecanismos vestibulares é de grande valia para a melhora das queixas de vertigem e tontura apresentadas por pacientes com sequelas de AVE. Porém, novos estudos devem ser realizados, com foco na avaliação da ansiedade, fatores ambientais relacionado, equilíbrio estático e dinâmico.

\section{REFERÊNCIAS}

ALBERTINO, S.; ALBERTINO, R.A. REABILITAÇÃO VESTIBULAR. Revista Otorrinolaringologia Geriátrica. 2012; 11(3).

CASTRO, Ana Sílvia Oliveira; GAZZOLA Juliana Marcia; NATOUR Jamil, et al. Versão brasileira do Dizziness Handicap Inventory. Pró-Fono Revista de Atualização Científica. V. 19, n.1, 97-104, 2007.

FERNANDES, Corina Aparecida; VASCONCELOS, Luciana Auxiliadora de Paula Vasconcelos. Reabilitação Vestibular após Acidente Vascular. Cerebral: Relato de Casos. Revista Neurociência. v.20, n.4, 560-566, 2012.

GANANÇA MM, Munhoz MSL, CAOVILLA HH, Silva MLG. Condutas na vertigem. São Paulo: Grupo Editorial Moreira Jr: 2004, 112p. 
GRILL, Eva; MÜLLER, M; BRANDT T, et al. Vertigo and dizziness: challenges for epidemiological research. OA Epidemiol. $2013 ; 1(2): 12$.

LACOUR, Michel; DEMANZE, Laurence. Interaction between vestibular compensation mechanisms and vestibular rehabilitation therapy: 10 recommendations for optimal functional recovery. Frontiers Neurology.v.5, 1-14, 2014.

MANTELLO, Erika Barioni; MORIGUT,I Julio Cesar; JUNIOR, Rodrigues, et al. Efeito da reabilitação vestibular sobre a qualidade de vida de idosos labirintopatas. Revista Brasileira de Otorrinolaringologia. v.74, n.2, 172-180, 2008.

MIYAMOTO, S. T. Brazilian version of the Berg balance scale. Braz. Med. Biol. Res., Ribeirão Preto. 2004; 37(9): 1411 1421.

PAVAN, Karina; LETKASKE Fernanda; SAKAMOTO, Tatiane, et al. Reabilitação vestibular em pacientes neurológicos. Medicina e Reabilitação. v.29, n.2,31-6, 2010.

PIRES Ana Paula Batista de Ávila, et al. Função Vestibular no acidente vascular cerebral do território carotídeo. Jornal Brasileiro de Otorrinolaringologia. v.79, n.1, 22-27, 2013. POLLOCK, Alex; HAZELTON, Christine; HENDERSON, Clair, et al. Interventions for disorders of eye movement in patients with stroke. Cochrane Database Systematic Reviews.v.5, n. 10,1-32, 2011

RIBEIRO, Angela dos Santos Bersot; PEREIRA, João Santos. Melhora do equilíbrio e redução da possibilidade de queda em idosas após os exercícios de Cawthorne e Cooksey. Rev Bras Otorrinol. V. 71, n.1, 38-46, 2005.

SALES, Renata. Achados de acuidade visual estática e dinâmica em pacientes com disfunção vestibular. $106 f$.
Tese (Doutorado) - Faculdade de Medicina de Ribeirão Preto, São Paulo, 2013.

SILVA, André Luis Santos; MOREIRA, João Soares. Vertigem: a abordagem da fisioterapia. Revista Fisioterapia Brasil. V.1, n.2, 91-97, 2000.

SIMONEAU, Guy; ULBRECHT, Jan; DERR, Janice; CAVANAGH Peter. Role of somatosensory input in the control of human posture. Gait Posture. v. 3, 115-22, 1995.

TEIXEIRA, Lázaro Juliano; MACHADO, João Natel Pollonio. Manobras para o tratamento da vertigem posicional paroxística benigna: revisão sistemática da literatura. Revista Brasileira Otorrinolaringologia. v.72, n.1,130-8, 2006.

TJERNSTROM Fredik, ZUR Oz, JAHN Klaus. Current concepts and future approaches to vestibular Rehabilitation. Journal Neurology. V. 263 (Suppl 1): S65-S70, 2016.

TOLEDO, Hernán; CORTÉS, Matheus Lopes; PANE, Christina, et al. Semont maneuver and vestibular rehabilitation exercises in the treatment of benign paroxysmal postural vertigo. A comparative study. Neurologia. V.15, n.4, 152-7, 2000.

WHITNEY Susan; ALGHADIR, Ahmad; ANWER, Shahnawaz. Recent evidence about the effectiveness of vestibular rehabilitation. Current Treatment Options in Neurology. V. 18, n.3, 13, 20016.

WYK, Andoret Van; EKSTEEN, Carina; RHEEDER, Paul. The effect of visual scanning exercises integrated into physiotherapy in patients with unilateral spatial neglect poststroke: a matched-pair randomized control trial. Neurorehabil Neural Repair. V.28, n.9, 856-73, 2014. 